\title{
Characteristics and Injury Mechanisms of Veteran Primary Care Suicide Decedents with and without Diagnosed Mental Illness
}

\author{
Joseph A. Simonetti, MD, MPH ${ }^{1,2,3}$, Rebecca Piegari, $M S^{4}$, Charles Maynard, $P h D^{5}$, \\ Lisa A. Brenner, $P h D^{7,6}$, Alaina Mori, BA ${ }^{5}$, Edward P. Post, MD, PhD 7,8 , \\ Karin Nelson, MD, MSHS $5,9,10$, and Ranak Trivedi, $P h D^{11,12}$
}

\begin{abstract}
'Rocky Mountain Mental Illness Research, Education and Clinical Center, Rocky Mountain Regional VA Medical Center, G3-189, 1700 N Wheeling St, Aurora, CO, USA; ${ }^{2}$ Denver-Seattle Center of Innovation for Veteran-Centered and Value-Driven Care, Veterans Health Administration, Washington, D.C., USA; ${ }^{3}$ Division of Hospital Medicine, University of Colorado Anschutz School of Medicine, Aurora, CO, USA; ${ }^{4}$ Department of Veterans Affairs, Clinical Systems Development and Evaluation, Veterans Health Administration, Washington, D.C., USA; ${ }^{5}$ VHA Primary Care Analytics Team, VA Puget Sound Healthcare System, Seattle, WA, USA; ${ }^{6}$ Departments of Physical Medicine and Rehabilitation, Psychiatry, and Neurology, University of Colorado Anschutz School of Medicine, Aurora, CO, USA; ${ }^{7}$ VA HSR\&D Center for Clinical Management Research, Ann Arbor, MI, USA; ${ }^{8}$ Department of Internal Medicine, University of Michigan Medical School, Ann Arbor, MI, USA; ${ }^{9}$ Department of Medicine, University of Washington School of Medicine, Seattle, WA, USA; ${ }^{10}$ General Medicine Service, VA Puget Sound Healthcare System, Seattle, WA, USA; ${ }^{11}$ Center for Innovation to Implementation, VA Palo Alto Health Care System, Palo Alto, CA, USA; ${ }^{12}$ Department of Psychiatry and Behavioral Sciences, Division of Public Mental Health and Population Sciences, Stanford University, Palo Alto, CA, USA.
\end{abstract}

BACKGROUND: In the United States, suicide rates are increasing among nearly all age groups. Primary care is a critical setting for suicide prevention, where interventions often rely on identifying mental health conditions as indicators of elevated suicide risk.

OBJECTIVE: Quantify the proportion of suicide decedents within primary care who had no antecedent mental health or substance use diagnosis.

DESIGN: Retrospective cohort study.

PARTICIPANTS: Veterans who received Veterans Health Administration (VHA) primary care any time from 2000 to 2014 and died by suicide before $2015(n=27,741)$.

MAIN MEASURES: We categorized decedents by whether they had any mental health or substance use diagnosis (yes/no) using ICD-9 codes available from VHA records. We compared sociodemographic, clinical, and suicide mechanism characteristics between groups using chisquare, Student's T, or Wilcoxon tests.

RESULTS: Forty-five percent of decedents had no mental health or substance use diagnosis. Decedents without such a diagnosis were older (68 vs. 57 years, $p<0.001$ ), and more likely to be male $(98.3 \%$ vs. $95.8 \%, p<0.001)$, non-Hispanic White (90.6\% vs. $87.9 \%, p<0.001)$, married/partnered (50.4\% vs. $36.6 \%, p<0.001)$, and without military service-connected disability benefits (72.6\% vs. $56.9 \%, p<0.001)$. They were also more likely to die from firearm injury $(78.9 \%$ vs. $60.7 \%, p<0.001)$. There were statistically significant differences in physical

Prior Presentation This work was presented as an oral abstract at the Society of General Internal Medicine Annual Meeting in Washington, D.C., in April 2019.

Electronic supplementary material The online version of this article (https://doi.org/10.1007/s11606-020-05787-1) contains supplementary material, which is available to authorized users.

Received November 12, 2019

Accepted March 9, 2020 health between groups, but the magnitudes of those differences were small. Decedents without a mental health or substance use diagnosis had significantly shorter durations of enrollment in VHA healthcare, less healthcare utilization in their last year of life, and had little utilization aside from primary care visits.

CONCLUSIONS AND RELEVANCE: From 2000 to 2014, of nearly thirty thousand VHA primary care patients who died by suicide, almost half had no antecedent mental health or substance use diagnosis. Within VHA primary care settings, suicide risk screening for those with and without such a diagnosis is indicated.

KEY WORDS: suicide; firearm; primary care; veteran.

$\mathrm{J}$ Gen Intern Med

DOI: $10.1007 / \mathrm{s} 11606-020-05787-1$

(C) Society of General Internal Medicine (This is a U.S. government work and not under copyright protection in the U.S.; foreign copyright protection may apply) 2020

\section{INTRODUCTION}

Suicide is the tenth leading cause of death in the United States (US). ${ }^{1}$ The suicide rate is approximately $20 \%$ higher among veterans in comparison with age and sex-matched nonveterans. ${ }^{2}$ Many suicide prevention interventions within the Veterans Health Administration (VHA) and elsewhere rely on identification and treatment of individuals with mental health conditions that are established risk factors for suicide (e.g., depression). ${ }^{4}$

Investigations conducted within non-VHA and military settings have found that $36-54 \%$ of those with suicidal behavior had no prior mental health diagnosis. ${ }^{3-7}$ Those studies relied on psychological autopsy to establish mental health diagnoses post-mortem, ${ }^{5}$ were conducted in systems without widespread mental health screening protocols, ${ }^{4,-8}$ or focused 
on commercially-insured ${ }^{3,9}$ or relatively young populations (most were under 50). 3, 4, 6, 7 Military servicemembers also have other important risk factors for suicide (e.g., physical and/or psychological traumas) and may underreport mental health issues due to privacy concerns. ${ }^{10,11}$

Understanding what proportion of veterans have recognized mental health or substance-related conditions prior to their suicide is critical for informing suicide risk screening strategies. Veterans have a higher prevalence of mental health and substance-related conditions in comparison with other US adults, and one-fourth of VHA primary care patients have established mental health or substancerelated conditions. ${ }^{12,13}$ However, of 968 veterans who died by suicide in Oregon from 2000 to $2005,57 \%$ had no recognized mental health condition prior to their death. ${ }^{14}$ In that study, investigators used state death certificate data to ascertain decedents' veteran status which is a method prone to misclassification. ${ }^{15}$ That study was also conducted before a significant expansion of VHA's mental health screening procedures.

Primary care is a critical setting for the identification and management of suicide risk. Nearly half of suicide victims have contact with a primary care provider within one month of their death and more than five million veterans are seen in VHA primary care clinics annually. ${ }^{16,17}$ However, within primary care populations, the proportion of suicide decedents who have mental health diagnoses prior to their death is unknown. The aim of this study was to quantify what proportion of suicide decedents who received primary care within VHA had antecedent mental health or substance use diagnosis, and compare characteristics between those with and without such diagnoses. Because suicide risk, risk factors, and methods differ between groups, we stratify analyses by race, sex, and ethnicity.

\section{METHODS}

\section{Study Design}

We conducted a retrospective cohort study of veterans who received VHA primary care from 2000 to 2014 and died by suicide before 2015. We used data from the VHA Corporate Data Warehouse (CDW) to identify all veterans who were assigned a primary care provider at any time during the study period. Using social security numbers, we identified those who died by suicide from the Department of Veterans Affairs (VA) and Department of Defense Joint Mortality Data Repository (MDR). ${ }^{18-20}$ The MDR includes information from the National Death Index (NDI), including cause of death classified by ICD-10 codes (suicide: $\mathrm{X} 60-\mathrm{X} 84, \mathrm{Y} 87.0) .{ }^{21}$ We excluded veterans whom we could not match to MDR records ( $0.1 \%$ of sample). Further details regarding matching veterans to NDI data are published elsewhere. ${ }^{19}$

\section{Mental Health Diagnoses}

Using ICD-9 codes available in the VHA CDW from 1999 to 2014, we categorized decedents by whether they had any mental health diagnosis (yes/no). Diagnoses of interest included depression, post-traumatic stress disorder (PTSD), anxiety disorder, serious mental illness (SMI; schizophrenia or bipolar disorder), or substance use disorder (SUD). Veterans were considered to have SUD if records revealed one inpatient or outpatient encounter that included a diagnostic code for a drug or alcohol use disorder (excluding tobacco use). All other diagnoses were considered present if relevant diagnostic codes were present during one inpatient or two outpatient encounters. We used different thresholds because VA administrative records have low sensitivity for identifying substance-related disorders in comparison with other mental health diagnoses. ${ }^{22,}$ 23

\section{Measures}

We abstracted sociodemographic characteristics from the CDW including age, sex, race, ethnicity, marital status, and percent service connection; a marker of military serviceconnected disability. We characterized each veteran's rurality (using Rural-Urban Continuum Codes) and US census region using address data, and county-level unemployment percentage using Bureau of Labor \& Statistics data. ${ }^{24,}{ }^{25}$ We calculated the Gagne comorbidity index for each veteran using ICD-9 codes. ${ }^{26}$ The Gagne index predicts one-year mortality based on the presence of certain mental and physical health diagnoses (e.g., malignancy, depression). ${ }^{26}$ Scores were calculated for decedents who had at least one Gagne condition. To assess physical health, we calculated a limited Gagne score in which we excluded mental health diagnoses. We used enrollment and encounter data to assess VHA enrollment duration and clinical encounters within one year of death. ${ }^{26}$ To assess recent changes in physical health, we identified Gagne conditions that were newly recorded within one year of death. Veterans have a high prevalence of traumatic brain injury (TBI), a condition associated with increased suicide risk. ${ }^{27-29}$ As such, a veteran was identified as having a history of TBI if relevant diagnostic codes were present during one inpatient or two outpatient encounters (ICD-9 codes 800.0 801.9, 803.0-804.9, 850.0-854.1). We identified mechanism of suicide (e.g., firearm) using ICD-10 codes available from the MDR.

\section{Statistical Analysis}

We summarized sociodemographic, clinical, and mechanism of death characteristics for the overall sample and compared differences between those with and without any mental health diagnosis using chi-square, Student's $T$, or Wilcoxon tests. We repeated analyses stratifying by sex, race, and ethnicity. Findings are reported consistent with Sex and Gender Equity in Research guidelines. ${ }^{30}$ We used SAS (version 9.4; SAS 
Table 1 Sociodemographic characteristics of VHA-enrolled suicide decedents with and without mental health diagnoses, 2000-2014

\begin{tabular}{|c|c|c|c|c|}
\hline & \multirow{2}{*}{$\frac{\text { Total }}{n=27,741}$} & \multirow{2}{*}{$\frac{\text { No MH diagnosis }}{n=12,480(45.0 \%)}$} & \multirow{2}{*}{$\frac{\text { MH diagnosis }}{n=15,261(55.0 \%)}$} & \multirow[t]{2}{*}{$p$ value } \\
\hline & & & & \\
\hline Age, mean years (SD) & $62(16)$ & $68(16)$ & $57(15)$ & $<0.001$ \\
\hline Age groups, $n(\%)$ & & & & $<0.001$ \\
\hline 18-34 years & 1906 & $561(4.5 \%)$ & $1345(8.8 \%)$ & \\
\hline $35-54$ years & 6818 & $1841(14.8 \%)$ & $4977(32.6 \%)$ & \\
\hline 55-64 years & 6448 & $2021(16.2 \%)$ & $4427(29.0 \%)$ & \\
\hline $65-79$ years & 7872 & $4543(36.4 \%)$ & $3329(21.8 \%)$ & \\
\hline $80+$ years & 4692 & $3511(28.1 \%)$ & $1181(7.7 \%)$ & \\
\hline Male sex, $n(\%)$ & 26,883 & $12,267(98.3 \%)$ & $14,616(95.8 \%)$ & $<0.001$ \\
\hline \multicolumn{4}{|l|}{ Race/ethnicity, $n(\%)$} & $<0.001$ \\
\hline Non-Hispanic, White & 19,938 & $8255(90.6 \%)$ & $11,683(87.9 \%)$ & \\
\hline Non-Hispanic, Black & 1164 & $423(4.6 \%)$ & $741(5.6 \%)$ & \\
\hline Non-Hispanic, other & 586 & $190(2.1 \%)$ & $396(3.0 \%)$ & \\
\hline Hispanic & 717 & $241(2.7 \%)$ & $476(3.5 \%)$ & \\
\hline \multicolumn{4}{|l|}{ Marital status, $n(\%)$} & $<0.001$ \\
\hline Married/partnered & 11,730 & $6183(50.4 \%)$ & $5547(36.6 \%)$ & \\
\hline Separated/widowed/divorced & 11,561 & $4673(38.1 \%)$ & $6888(45.4 \%)$ & \\
\hline Never married & 4134 & $1407(11.5 \%)$ & $2727(18.0 \%)$ & \\
\hline \multicolumn{4}{|l|}{ Service connection, $n(\%)$} & $<0.001$ \\
\hline None & 17,735 & $9056(72.6 \%)$ & $8679(56.9 \%)$ & \\
\hline$<50 \%$ & 5283 & $2386(19.1 \%)$ & $2897(19.0 \%)$ & \\
\hline$\geq 50 \%$ & 4719 & $1034(8.3 \%)$ & $3685(24.2 \%)$ & \\
\hline Local unemployment rate, mean \% (SD) & $7.0(2.8)$ & $6.9(2.7)$ & $7.1(2.8)$ & $<0.001$ \\
\hline \multicolumn{4}{|l|}{ US Census region, $n(\%)$} & $<0.001$ \\
\hline Northeast & 3103 & $1421(11.5 \%)$ & $1682(11.2 \%)$ & \\
\hline Midwest & 5726 & $2576(20.9 \%)$ & $3150(20.9 \%)$ & \\
\hline South & 11,616 & $5336(43.2 \%)$ & $6280(41.6 \%)$ & \\
\hline \multirow{2}{*}{\multicolumn{4}{|c|}{ Rurality, $n(\%)$}} & \\
\hline & & & & $<0.001$ \\
\hline Rural & 2122 & $979(7.9 \%)$ & $1143(7.5 \%)$ & \\
\hline Urban & 10,712 & $4663(37.6 \%)$ & $6049(39.9 \%)$ & \\
\hline Suburban & 14,743 & $6757(54.5 \%)$ & $7986(52.6 \%)$ & \\
\hline
\end{tabular}

Abbreviations: VHA, Veterans Health Administration; MH, mental health; US, United States

Denominators for column percentages include only those with non-missing data

Institute, Inc., Cary, NC) for all analyses. These evaluation efforts are part of ongoing quality improvement efforts at VHA and are not considered research activity or subject to institutional review board review or waiver.

\section{RESULTS}

Of 9,550,726 veterans enrolled in VHA primary care from 2000 to $2014,2,724,002$ died (28.5\%) and 27,741 (1.0\%) died by suicide. Of those, 15,261 (55.0\%) met criteria for at least one mental health or substance use diagnosis (Table 1).

The mean age of the suicide decedent sample was 62 years, $96.9 \%$ were male, $89.0 \%$ were non-Hispanic White, and $42.8 \%$ were married or partnered (Table 1). Thirty-six percent of the sample had service-connected disability benefits, and $7.7 \%$ and $53.5 \%$ lived in rural and suburban areas, respectively. In comparison with those who had a mental health or substance use diagnosis, decedents without such a history were more likely to be older ( 68 vs. 57 years, $p<0.001$ ), male $(98.3 \%$ vs. $95.8 \%, p<0.001)$, non-Hispanic White $(90.6 \%$ vs. $87.9 \%, p<0.001)$, married or partnered $(50.4 \%$ vs. $36.6 \%$, $p<0.001)$, and without disability benefits $(72.6 \%$ vs. $56.9 \%$, $p<0.001)$. Although statistically significant, only small differences were observed between groups in geographic characteristics (e.g., unemployment rate, rurality).
Of those who had at least one mental health or substance use diagnosis, $73.2 \%$ had a depressive disorder, 25.5\% had PTSD, $32.9 \%$ had an anxiety disorder, $22.0 \%$ had an SMI, and $50.4 \%$ had an SUD (Table 2). Those without mental health diagnoses were less likely to have a history of TBI $(0.4 \%$ vs. $2.5 \%$, $p<0.001)$ and at least one Gagne comorbidity condition $(57.5 \%$ vs. $86.2 \%, p<0.001)$. Among those with at least one Gagne condition, decedents without a mental health or substance use diagnosis had lower Gagne comorbidity scores $(0.8$ vs. $1.1, p<0.001)$ and fewer total Gagne conditions (2.7 vs. $3.4, p<0.001)$. Decedents without a mental health or substance use diagnosis had higher limited Gagne comorbidity scores $(0.8$ vs. $0.7, p<0.01)$ in which only physical health diagnoses were considered. In the year prior to death, decedents without a mental health or substance use diagnosis were less likely to be diagnosed with a Gagne condition $(34.7 \%$ vs. $56.9 \%, p<0.001)$ or physical health Gagne condition $(31.8 \%$ vs. $41.2 \%, p<0.001)$.

In comparison with those who had a mental health or substance use diagnosis, decedents without such a history had significantly shorter durations of enrollment in VHA (7.1 vs. 7.7 years, $p<0.001)$ and VHA primary care ( 4.0 vs. 5.0 years, $p<0.001)$. In their last year of life, they had significantly fewer primary care ( 2.3 vs. 4.3$)$, mental health ( 0.1 vs. 7.7), and emergency department visits (0.2 vs. 0.6$)$, and were 
Table 2 Clinical characteristics and healthcare utilization of VHA-enrolled suicide decedents with and without mental health diagnoses, 20002014

\begin{tabular}{|c|c|c|c|c|}
\hline & \multirow{2}{*}{$\begin{array}{l}\text { Total } \\
n=27,741\end{array}$} & \multirow{2}{*}{$\frac{\text { No MH diagnosis }}{n=12,480(45.0 \%)}$} & \multirow{2}{*}{$\begin{array}{l}\text { MH diagnosis } \\
n=15,261(55.0 \%)\end{array}$} & \multirow[t]{2}{*}{$p$ value } \\
\hline & & & & \\
\hline \multicolumn{5}{|l|}{ Mental health diagnoses, $n(\%)$} \\
\hline Depression & 11,171 & - & $11,171(73.2 \%)$ & \\
\hline PTSD & 3886 & - & $3886(25.5 \%)$ & \\
\hline Anxiety disorder & 5014 & - & $5014(32.9 \%)$ & \\
\hline SMI & 3354 & - & $3354(22.0 \%)$ & \\
\hline SUD & 7692 & - & $7692(50.4 \%)$ & \\
\hline \multicolumn{5}{|l|}{ Comorbidities } \\
\hline TBI, $n(\%)$ & 428 & $48(0.4 \%)$ & $380(2.5 \%)$ & $<0.001$ \\
\hline Patients with at least 1 Gagne condition, $n(\%)$ & $20,329(73.3 \%)$ & $7172(57.5 \%)$ & $13,157(86.2 \%)$ & $<0.001$ \\
\hline Gagne comorbidity score, mean (SD) ${ }^{\mathrm{a}}$ & $0.97(1.85)$ & $0.83(1.98)$ & $1.05(1.76)$ & $<0.001$ \\
\hline Gagne limited comorbidity score, ${ }^{b}$ mean (SD) & $0.78(1.90)$ & $0.84(1.99)$ & $0.74(1.83)$ & $<0.01$ \\
\hline Total Gagne conditions, mean (SD) & $3.17(2.03)$ & $2.68(1.76)$ & $3.44(2.11)$ & $<0.001$ \\
\hline Total limited Gagne conditions, ${ }^{\mathrm{b}}$ mean (SD) & $2.62(1.77)$ & $2.64(1.72)$ & $2.61(1.80)$ & 0.24 \\
\hline \multicolumn{5}{|l|}{ Prior year new diagnoses by Gagne conditions } \\
\hline Prior year new Gagne diagnoses, $n(\%)$ & $13,013(46.9 \%)$ & $4336(34.7 \%)$ & $8677(56.9 \%)$ & $<0.001$ \\
\hline Prior year new limited Gagne diagnoses, ${ }^{a} n(\%)$ & $10,268(37.0 \%)$ & $3974(31.8 \%)$ & $6294(41.2 \%)$ & $<0.001$ \\
\hline Mental health & $5496(19.8 \%)$ & $756(6.1 \%)$ & $4740(31.1 \%)$ & $<0.001$ \\
\hline Pulmonary disorder & $1787(6.4 \%)$ & $670(5.4 \%)$ & $1117(7.3 \%)$ & $<0.001$ \\
\hline Malignancy & $1197(4.3 \%)$ & $667(5.3 \%)$ & $530(3.5 \%)$ & $<0.001$ \\
\hline Cardiac disease & $2766(10.0 \%)$ & $1209(9.7 \%)$ & $1557(10.2 \%)$ & 0.16 \\
\hline Neurologic disease & $1410(5.1 \%)$ & $505(4.0 \%)$ & $905(5.9 \%)$ & $<0.001$ \\
\hline Diabetes & $1290(4.7 \%)$ & $594(4.8 \%)$ & $696(4.6 \%)$ & 0.44 \\
\hline Renal & $500(1.8 \%)$ & $245(2.0 \%)$ & $255(1.7 \%)$ & 0.07 \\
\hline Rheumatologic & $186(0.7 \%)$ & $66(0.5 \%)$ & $120(0.8 \%)$ & 0.01 \\
\hline Dementia & $139(0.5 \%)$ & $34(0.3 \%)$ & $105(0.7 \%)$ & $<0.001$ \\
\hline Liver disease & $587(2.1 \%)$ & $106(0.8 \%)$ & $475(3.1 \%)$ & $<0.001$ \\
\hline Miscellaneous $^{c}$ & $5299(19.1 \%)$ & $1995(16.0 \%)$ & $3304(21.6 \%)$ & $<0.001$ \\
\hline Length of VHA enrollment, mean years (SD) & $7.5(4.2)$ & $7.1(4.2)$ & $7.7(4.2)$ & $<0.001$ \\
\hline Length of primary care enrollment, mean years (SD) & $4.5(3.4)$ & $4.0(3.3)$ & $5.0(3.4)$ & $<0.001$ \\
\hline \multicolumn{5}{|l|}{ Prior year utilization } \\
\hline Primary care visits, mean (SD) & $3.4(4.7)$ & $2.3(3.6)$ & $4.3(5.3)$ & $<0.001$ \\
\hline Mental health visits, mean (SD) & $4.3(14.1)$ & $0.1(1.0)$ & $7.7(18.3)$ & $<0.001$ \\
\hline Emergency department visits, mean (SD) & $0.4(1.3)$ & $0.2(0.8)$ & $0.6(1.7)$ & $<0.001$ \\
\hline Hospitalizations, mean (SD) & $0.3(0.8)$ & $0.1(0.4)$ & $0.4(1.0)$ & $<0.001$ \\
\hline Any hospitalization, $n(\%)$ & $3771(13.6 \%)$ & $643(5.2 \%)$ & $3128(20.5 \%)$ & $<0.001$ \\
\hline
\end{tabular}

Abbreviations: VHA, Veterans Health Administration; MH, mental health; PTSD, post-traumatic stress disorder; SMI, serious mental illness; SUD, substance use disorder; TBI, traumatic brain injury; SD, standard deviation

${ }^{a}$ Gagne score only calculated among patients with at least one Gagne condition

${ }^{b}$ Limited Gagne score excludes depression, psychosis, and drug and alcohol-related conditions

${ }^{c}$ Includes various conditions, such as hypertension, electrolyte abnormalities, anemia, weight loss, human immunodeficiency virus infection, ulcer, and coagulopathy

Denominators for column percentages include only those with non-missing data

less likely to be hospitalized (5.2\% vs. $20.5 \%)(p<0.001$ all comparisons, Table 2).

In stratified analyses, $19.8 \%$ of 803 female decedents and $45.6 \%$ of 26,883 male decedents had no history of a mental health or substance use diagnosis (ESM Tables 1a-b). In comparison with females who had a mental health or substance use diagnosis, female decedents without such a history had significantly lower Gagne comorbidity scores $(0.1$ vs. 0.8 , $p<0.001)$ and fewer Gagne conditions (1.8 vs. 3.1, $p<0.001$ ) (overall and limited to physical health diagnoses). They were also significantly less likely to receive a new mental or physical health diagnosis in their last year of life ( $23.3 \%$ vs. $51.7 \%$, $p<0.001)$. In comparison with females who had a mental health or substance use diagnosis, female decedents without such a history had significantly shorter durations of enrollment in VHA (5.9 vs. 7.8 years, $p<0.001)$ and VHA primary care ( 2.8 vs. 5.0 years, $p<0.001$ ). In the year prior to death, female decedents without a mental health or substance use diagnosis had significantly fewer primary care (1.5 vs. 5.6), mental health (0.3 vs. 12.7$)$, and emergency department visits $(0.2$ vs. 0.9$)$, and were less likely to be hospitalized ( $2.5 \%$ vs. $25.5 \%)(p<0.001$ all comparisons).

Forty-one percent of non-Hispanic White decedents, 36.3\% of non-Hispanic Black decedents, $32.4 \%$ of non-Hispanic decedents of other races, and $33.6 \%$ of Hispanic decedents did not have a mental health or substance use diagnosis prior to their death (ESM Tables 2a-d). Additional comparisons among racial and ethnic groups are shown in ESM Tables 2a-d.

Of 27,741 suicide decedents, $68.9 \%$ died by firearm injury; $13.0 \%$ died by poisoning; $12.1 \%$ died from hanging, strangulation, or suffocation; and less than $5 \%$ died by drowning, jumping from a height, or laceration (Fig. 1). In comparison with those who had a mental health or substance use diagnosis, decedents without such a history were more likely to die by firearm injury among the full sample $(78.9 \%$ vs. $60.7 \%$, 


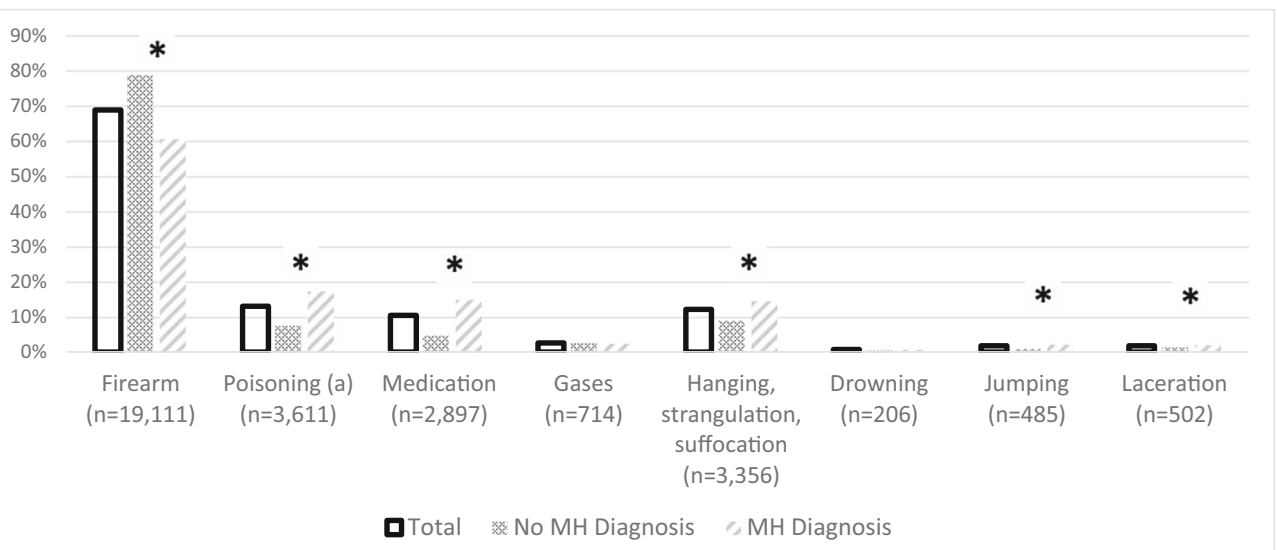

Figure 1 Injury mechanisms of VHA-enrolled suicide decedents with and without mental health diagnoses, 2000-2014. VHA Veterans Health Administration, MH mental health. Denominators for column percentages include only those with non-missing data. (a) Medications and gases are subcategories of poisoning. * $p$ value $<\mathbf{0 . 0 5}$, comparing suicide mechanism between those with and without diagnosed mental health conditions.

$p<0.001$ ), both sexes, and all races and ethnicities (ESM Tables 1c-d, 2e-h).

\section{CONCLUSIONS}

From 2000 to 2014, of nearly thirty thousand veteran primary care patients who died by suicide, almost half had no evidence of an antecedent mental health or substance use diagnosis. Despite differences in data sources, populations, healthcare systems, and time periods, this estimate is similar to those reported in prior studies. ${ }^{5,28,}{ }^{31}$ Notably, the proportion of decedents who had a mental health diagnosis was substantially higher among female in comparison with male veterans ( $80.2 \%$ vs. $54.4 \%)$. Among the full sample, both sexes, and all races and ethnicities, veterans without a mental health diagnosis were more likely to die from firearm injury.

Nearly half of all suicide decedents had no mental health diagnosis, and those without such a diagnosis had lower healthcare utilization and little utilization aside from that received in primary care. These findings have important implications for suicide prevention strategies within primary care. VHA is expanding its screening procedures to assess suicidal ideation among veterans seeking care in outpatient, inpatient, and specialty care settings regardless of their mental health history. ${ }^{32}$ A key question is whether this initiative will be successful in identifying and intervening with at-risk veterans who would not have come to attention through routine mental health and substance use screening procedures. Future research could also be helpful in determining whether more frequent screening or screenings using different methodologies (e.g., telehealth, phone visits) might identify more at-risk individuals.

Suicide decedents without a mental health or substance use diagnosis were more likely to be male, older, non-Hispanic White, married or partnered, and without service-connected disabilities. These findings are consistent with a 2015 investigation of nearly 21,000 suicide decedents from 27 US states. ${ }^{5}$ Those without mental health conditions were more likely to be male, older, and have served in the military. Not having a service-connected disability has practical implications within VHA, as those veterans bear a greater financial responsibility for their healthcare. Some of the sociodemographic differences between groups could in part be explained by disparities in screening practices or performance of screening measures among racial, ethnic, or sex groups. That is, veterans may have been miscategorized as lacking a diagnosis if screening or diagnostic measures underperform or are underutilized among their specific group. ${ }^{33}$

Suicide decedents with mental health histories had a sixfold higher prevalence of TBI; a condition associated with elevated suicide risk and other mental health and substance use conditions. ${ }^{34,35}$ Aside from this finding, we observed statistically significant, but overall small differences between groups in physical health, including comorbidity risk scores and prevalences of specific disease categories.

The physical health differences we observed between groups are unlikely to solely account for the suicide risk among those without mental health histories, nor are each of the physical diagnoses we identified clearly associated with increased suicide risk (e.g., anemia). Notably, those with a mental health history had substantially greater healthcare utilization in their last year of life, providing more opportunities for physical and mental health conditions to be identified. Forty-one percent of those with mental health histories received a physical health diagnosis in their last year of life in comparison with $32 \%$ of those without such histories. An important question for future investigations is whether physical health diagnoses contribute differentially to suicide risk among those with mental illness, or among specific sexes, races, or ethnicities.

Consistent with other studies, suicide decedents without a mental health diagnosis were substantially more likely to die 
from a firearm injury in comparison with those with such a diagnosis. ${ }^{5,}{ }^{28}$ This relationship was observed among both sexes and all races and ethnicities. However, a higher proportion of non-Hispanic White veterans died from firearm injury $(69.4 \%)$ in comparison with decedents of other races and those of Hispanic ethnicity. Firearm access is an independent risk factor for suicide. ${ }^{36,37}$ Some of the suicide risk among those without mental illness could be explained by a greater prevalence of firearm access. However, it seems unlikely that differences in firearm access solely explain this finding. A prior study found that those dying by firearm suicide have different physical health histories than those dying by other means. ${ }^{3}$ Taken together, these findings may suggest the presence of different suicide risk phenotypes, some of which may be in part defined by the presence of certain physical health diagnoses, personal and environmental stressors, and access to specific lethal means. Importantly, the VA's National Strategy for Preventing Veteran Suicide recommends addressing firearm access among "at-risk" veterans. ${ }^{38}$ Considering that those with elevated suicide risk may not be identified as such, our findings suggest that it may be important to address firearm-related suicide risk among a broader population.

Future work is needed to improve our understanding of factors driving suicide risk among primary care patients, particularly those who may not be identified as at-risk by routine mental health screenings. Prior work investigating suicide risk factors among Army servicemembers has shown that $84 \%$ of decedents had at least one "environmental stressor" prior to their death, including legal or relationship problems, abuse victimization or perpetration, and work stressors. ${ }^{4}$ Other investigations have identified similar stressors prior to suicide, stressors that were slightly more prevalent among decedents without mental health histories. ${ }^{5}$ Of those without a mental health history who received postmortem toxicology testing in one study, $71 \%$ tested positive for at least one substance (e.g., marijuana). Collecting similar stressor and toxicologic data will be critical for future research aiming to explain suicide risk among those without mental illness.

VHA must consider that certain diagnoses may increase suicide risk (e.g., malignancy) and draw patients into specialty care (i.e., oncology, palliative care), and possibly away from mental health or primary care services. ${ }^{39}$ For example, $5 \%$ of decedents in our sample without mental health diagnoses received a new malignancy diagnosis within one year of their death. Prior studies have shown that veterans living with cancer have an elevated risk of suicidal ideation, suicide, and firearm-related suicide. ${ }^{40,}{ }^{41}$ In some cases, given increasing opportunities for veterans to access community-based services, ${ }^{42}$ such diagnoses may even draw patients away from VHA-centric suicide prevention programs. Additional work is needed to evaluate the obstacles and impact of expanding suicide risk screening to other clinical settings, including those in non-VHA settings.
In comparison with prior work, this study benefited from the availability of diagnostic information from the health record, a large sample size, and NDI information which is considered the gold standard for ascertaining vital status and cause of death. ${ }^{4,5,28}$ However, there are limitations of this study. First, we may have miscategorized individuals as lacking a mental health diagnosis if they received care outside of the VHA system. Second, we categorized veterans as having a mental health diagnosis using all data within the record which may not reflect their actual risk profile just prior to their death. For example, administrative data may not capture severity of illness, and a veteran diagnosed with depression in 2004 may not have depressive symptoms in 2010. This approach was taken because even remote mental health diagnoses are risk factors for future suicide. Further, if this approach miscategorized veterans with respect to their suicide risk just prior to death (i.e., because they have symptom remission), our estimate of the proportion of veterans without mental illness would be an underestimate. Third, we limited the conditions included within the Gagne index to physical health conditions which may limit generalizability to other cohorts with respect to physical health comparisons. Fourth, the bivariate relationships presented in this study may be confounded by other factors.

Nearly half of VHA primary care patients who died by suicide from 2000 to 2014 did not have a mental health or substance use diagnosis, and those decedents had limited healthcare utilization aside from primary care services in their last year of life. These findings suggest that suicide prevention efforts relying solely on the identification of mental illness as an indicator for suicide risk may be insufficient in preventing a large proportion of suicides among veterans. Additional work is needed to better understand factors driving suicide risk among this group, why they are more likely to use firearms in their death, and how to identify individuals without mental illness who are at risk of suicide.

Corresponding Author: Joseph A. Simonetti, MD, MPH; Division of Hospital Medicine University of Colorado Anschutz School of Medicine, Aurora, CO, USA (e-mail: joseph.simonetti@ucdenver.edu).

Funding Information This work was undertaken as part of the VHA Primary Care Analytics Team (PCAT), funded by the VA Office of Primary Care. The funding office had no role in the design, conduct, or interpretation of this study or in the decision to submit this manuscript for publication.

\section{Compliance with Ethical Standards:}

Conflict of Interest: The authors have no conflicts of interest to disclose.

Disclaimer: This work was undertaken as part of the VHA Primary Care Analytics Team (PCAT), funded by the VA Office of Primary Care. The funding office had no role in the design, conduct, or interpretation of this study or in the decision to submit this manuscript for publication. Data for this report were developed by PCAT. The views expressed are those of the authors and do not necessarily reflect the position or policy of the Department of Veterans Affairs. 


\section{REFERENCES}

1. CDC. Web-Based Injury Statistics Query \& Reporting System (WISQARS), Fatal injury reports. National Center for Injury Prevention and Control Centers for Disease Control and Prevention. Available at: http://www.cdc. gov/injury/wisqars/fatal.html. Accessed June 27 2019. 2019.

2. VHA Office of Suicide Prevention. Suicide among Veterans and other Americans, 2001-2014. 2016. Available at: www.mentalhealth.va.gov/ docs/2016suicidedatareport.pdf. Accessed 26 June 2019.

3. Boggs JM, Beck A, Hubley S, et al. General Medical, Mental Health, and Demographic Risk Factors Associated With Suicide by Firearm Compared With Other Means. Psychiatr Serv. 2018;69(6):677-684.

4. Black SA, Gallaway MS, Bell MR, Ritchie EC. Prevalence and Risk Factors Associated With Suicides of Army Soldiers 2001-2009. Military Psychology. 2011;23(4):433-451.

5. Stone DM, Simon TR, Fowler KA, et al. Vital Signs: Trends in State Suicide Rates - United States, 1999-2016 and Circumstances Contributing to Suicide - 27 States, 2015. MMWR Morb Mortal Wkly Rep 2018;67:617-624. https://doi.org/10.15585/mmwr.mm6722al. Accessed January 232020.

6. Ursano RJ, Kessler RC, Naifeh JA, et al. Risk Factors Associated With Attempted Suicide Among US Army Soldiers Without a History of Mental Health Diagnosis. JAMA Psychiatry. 2018;75(10):1022-1032.

7. Naifeh JA, Ursano RJ, Kessler RC, et al. Suicide attempts among activated soldiers in the U.S. Army reserve components. BMC psychiatry. 2019;19(1):31-31.

8. Reger MA, Smolenski DJ, Carter SP. Suicide prevention in the us army: A mission for more than mental health clinicians. JAMA Psychiatry. 2018;75(10):991-992

9. Description of MHRN Denominator Population 2009-2011. Mental Health Research Network. Availble at: http://hcsrn.org/mhrn/en/ Tools\%20\&\%20Materials/MHRNResources/MHRN_Denominator.pdf. Accessed May 142019

10. Vannoy SD, Andrews BK, Atkins DC, Dondanville KA, Young-McCaughan $\mathrm{S}$, Peterson AL. Under Reporting of Suicide Ideation in US Army Population Screening: An Ongoing Challenge. Suicide Life Threat Behav. 2017;47(6):723-728.

11. Ursano RJ, Kessler RC, Naifeh JA, et al. Associations of Time-Related Deployment Variables With Risk of Suicide Attempt Among Soldiers: Results From the Army Study to Assess Risk and Resilience in Servicemembers (Army STARRS). JAMA Psychiatry. 2018;75(6):596-604

12. Trivedi RB, Post EP, Sun H, et al. Prevalence, Comorbidity, and Prognosis of Mental Health Among US Veterans. Am J Public Health. 2015;105(12):2564-2569.

13. Hoerster KD, Lehavot K, Simpson T, McFall M, Reiber G, Nelson KM. Health and Health Behavior Differences. American Journal of Preventive Medicine. 2012;(43):483-489.

14. Basham C, Denneson LM, Millet L, Shen X, Duckart J, Dobscha SK Characteristics and VA health care utilization of U.S. Veterans who completed suicide in Oregon between 2000 and 2005. Suicide Life Threat Behav. 2011;41(3):287-296.

15. US Department of Veterans Affairs. Report of the blue ribbon work group report on suicide prevention in the veteran population. 2008. Available at: http://www.mentalhealth.va.gov/suicide_prevention/Blue_Ribbon_Report-FINAL June-30-08.pdf. Accessed January 232020.

16. Luoma JB, Martin CE, Pearson JL. Contact with mental health and primary care providers before suicide: a review of the evidence. Am J Psychiatry. 2002;159(6):909-916.

17. Rosland AM, Nelson K, Sun H, et al. The patient-centered medical home in the Veterans Health Administration. Am J Manag Care. 2013;19(7):e263-272.

18. Maynard C. Ascertaining veterans' vital status: VA data sources for mortality ascertainment and cause of death. VIReC Database \& Methods Cyberseminar series. 2019. Available at: https://www.hsrd.research.va. gov/for_researchers/cyber_seminars/archives/video_archive.cfm? SessionID=3544. Accessed May 142019.

19. Bossarte R, Schneiderman A. An Overview of VA/DoD Joint Mortality Data Repository and Data From Studies Conducted by the Epidemiology Program in VA's Office of Public Health. VA HSR\&D Cybersminar. 2015 Available at: https://www.hsrd.research.va.gov/for_researchers/cyber_ seminars. Accessed May 82019.

20. Center of Excellence for Suicide Prevention. Joint Department of Veterans Affairs (VA) and Department of Defense (DoD) Suicide Data Repository
(SDR) - National Death Index (NDI). Available at: http://www.dspo.mil/ Portals/113/Documents/SDR\%20Fact\%20Sheet.pdf. Accessed February 24, 2017.

21. National Death Index. Division of Vital Statistics, National Center for Health Statistics, Center for Disease Control and Prevention (CDC). Atlanta, GA. 2016. Available at: https://www.cdc.gov/nchs/ndi/index. htm. Accessed February 5, 2018.

22. Kashner TM. Agreement between administrative files and written medical records: a case of the Department of Veterans Affairs. Med Care. 1998;36(9):1324-1336.

23. Kim HM, Smith EG, Stano CM, et al. Validation of key behaviourally based mental health diagnoses in administrative data: suicide attempt, alcohol abuse, illicit drug abuse and tobacco use. BMC Health Serv Res. 2012;12:18.

24. US Department of Labor, Bureau of Labor Statistics. State and local unemployment rates. Available at: https://www.bls.gov/lau/. Accessed February 5, 2018.

25. Rural-Urban Continuum Codes, 2013. U.S. Department of Agriculture Available at: https://www.ers.usda.gov/data-products/rural-urban-continuum-codes.aspx\#.UYJuVEpZRvY). Accessed January 212020.

26. Gagne JJ, Glynn RJ, Avorn J, Levin R, Schneeweiss S. A combined comorbidity score predicted mortality in elderly patients better than existing scores. Journal of clinical epidemiology. 2011;64(7):749-759.

27. Madsen T, Erlangsen A, Orlovska S, Mofaddy R, Nordentoft M, Benros ME. Association between traumatic brain injury and risk of suicide. JAMA. 2018;320(6):580-588.

28. Boggs JM, Simon GE, Ahmedani BK, Peterson E, Hubley S, Beck A. The Association of Firearm Suicide With Mental Illness, Substance Use Conditions, and Previous Suicide Attempts. Ann Intern Med. 2017;167(4):287-288

29. Ahmedani BK, Peterson EL, Hu Y, et al. Major Physical Health Conditions and Risk of Suicide. Am J Prev Med. 2017;53(3):308-315.

30. Heidari S, Babor TF, De Castro P, Tort S, Curno M. Sex and Gender Equity in Research: rationale for the SAGER guidelines and recommended use. Research Integrity and Peer Review. 2016;1(1):2.

31. Harris EC, Barraclough B. Suicide as an outcome for mental disorders. A meta-analysis. The British journal of psychiatry : the journal of mental science. 1997; 170:205-228.

32. Young, Steve. Suicide risk screening and assessment requirements. Deputy Under Secretary for Health for Operations and Management (10N), Department of Veterans Affairs. Memorandum, May 23, 2018.

33. Hahm HC, Cook BL, Ault-Brutus A, Alegria M. Intersection of raceethnicity and gender in depression care: screening, access, and minimally adequate treatment. Psychiatr Serv. 2015;66(3):258-264.

34. Bahraini NH, Simpson GK, Brenner LA, Hoffberg AS, Schneider AL Suicidal Ideation and Behaviours after Traumatic Brain Injury: A Systematic Review. Brain Impairment. 2013;14(1):92-112.

35. Greer N, Sayer NA, Spoont M, et al. Prevalence and Severity of Psychiatric Disorders and Suicidal Behavior in Service Members and Veterans With and Without Traumatic Brain Injury: Systematic Review. The Journal of head trauma rehabilitation. 2019

36. Miller M, Barber C, White RA, Azrael D. Firearms and Suicide in the United States: Is Risk Independent of Underlying Suicidal Behavior? American Journal of Epidemiology. 2013;178(6):946-955.

37. Miller M, Swanson SA, Azrael D. Are We Missing Something Pertinent? A Bias Analysis of Unmeasured Confounding in the Firearm-Suicide Literature. Epidemiologic reviews. 2016;38(1):62-69.

38. US Department of Veterans Affairs. National Strategy for Preventing Veteran Suicide, 2018-2028. Available at: https://www.mentalhealth.va. gov/suicide_prevention/docs/Office-of-Mental-Health-and-Suicide-Prevention-National-Strategy-for-Preventing-Veterans-10.1007/s11606020-05787-1Suicide.pdf. Accessed August 12, 2019.

39. Massetti GM, Holland KM, Jack SPD, Ragan KR, Lunsford NB. Circumstances of suicide among individuals with a history of cancer. Psychooncology. 2018;27(7):1750-1756.

40. Johnson CC, Phillips KM, Miller SN. Suicidal Ideation among Veterans Living with Cancer Referred to Mental Health. Clinical gerontologist. 2020;43(1):24-36.

41. Sullivan DR, Forsberg CW, Golden SE, Ganzini L, Dobscha SK, Slatore CG. Incidence of Suicide and Association with Palliative Care among Patients with Advanced Lung Cancer. Annals of the American Thoracic Society. 2018;15(11):1357-1359.

42. US Department of Veterans Affairs. VA Mission Act. Available at: https:// missionact.va.gov/. Accessed June 10, 2019.

Publisher's Note: Springer Nature remains neutral with regard to jurisdictional claims in published maps and institutional affiliations. 